\title{
Acknowledgement to Reviewers of Journal of Functional Biomaterials in 2017
}

\author{
Journal of Functional Biomaterials Editorial Office \\ MDPI AG, St. Alban-Anlage 66, 4052 Basel, Switzerland \\ Published: 12 January 2018
}

Peer review is an essential part in the publication process, ensuring that Journal of Functional Biomaterials maintains high quality standards for its published papers. In 2017, a total of 52 papers were published in the journal. Thanks to the cooperation of our reviewers, the median time to first decision was 18 days and the median time to publication was 43 days. The editors would like to express their sincere gratitude to the following reviewers for their time and dedication in 2017:

\author{
Akhavan, Behnam \\ Al-Dujaili, Emad \\ Alem, Halima \\ Amorati, Riccardo \\ Aniello, Francesco \\ Arnaud, Françoise \\ Atrens, Andrej \\ Biglino, Giovanni \\ Bouropoulos, Nikolaos \\ Brantley, William A. \\ Brauer, Jeremy A. \\ Busquets, Maria Antònia \\ Calvo-Guirado, José Luis \\ Capasso, Raffaele \\ Cervadoro, Antonio \\ Chang, Thomas M.S. \\ Chang, Wen-Wei \\ Chou, Joshua \\ Cicciu, Marco \\ Collins, Maurice N. \\ Coltelli, Maria Beatrice \\ Costa-Rodrigues, João \\ Cristea, Cecilia \\ De Aza, Piedad \\ Do, Sun Hee \\ Doloff, Joshua C. \\ Eggenhöffner, Roberto \\ Esposito, Ciro \\ Farsad, Khashayar \\ Fauzi, Iliana \\ Fernandes, Susana C.M. \\ Feyerabend, Frank \\ Figueiredo, Marxa
}

\author{
Kramer, Phillip \\ Kundu, Joydip \\ Lebaron, Richard \\ Lee, Hae-Hyoung \\ Lee, Wonhye \\ Lee, WooCheol \\ Llena, Carmen \\ Łukomksa-Szymańska, Monika \\ Madeddu, Roberto \\ Maeda, Hirotaka \\ Mahapatro, Anil \\ Mandava, Nanda \\ Mao, Chuanbin \\ Marpu, Sreekar Babu \\ Martin, Thomas John \\ Martins, Albino \\ Maruyama, Kei \\ Mathew, Mathew \\ Mayer, Christian \\ McCoy, Sarah J. Breese \\ Mekonnen, Tizazu H. \\ Melo, Mary Anne S. \\ Menabue, Ledi \\ Mendes, Ana C. \\ Mizusaki, Masanobu \\ Naito, Yoshihito \\ Noubissi, Felicite \\ Oh, Daniel S. \\ Okamoto, Masami \\ Oskouei, Reza \\ Palumbo, Fabio Salvatore \\ Papini, Alessio \\ Partonen, Timo
}


Finley, Brent L.

Forner, Leopoldo

Gentile, Piergiorgio

Girousi, Stella

Gizdavic-Nikolaidis, Marija R.

Glaser, Rainer

Golkowski, Mark

Gómez-Florit, Manuel

Gracia, Ignacio

Grandfield, Kathryn

Granet, Robert

Graziani, Gabriela

Grenho, Liliana

Haapasalo, Markus

Hadjiargyrou, Michael

Hayakawa, Tohru

Herndon, David

Hildreth, Blake

Hirner, Alfred V.

$\mathrm{Hu}$, Jack

$\mathrm{Hu}$, Yang

Intaglietta, Marcos

Irie, Masao

Jennings, Jessica

Kevadiya, Bhaveah

Khandaker, Morshed

Kim, Chun Ho

Kim, Hyoung Seop

Kim, Kyung-su

Kim, Tae Im

Kim, Young-Jin

Knipping, Karen

Koizumi, Hiroyasu

Kootala, Sujit

Koźlecki, Tomasz
Patterson, Jennifer

Penarrocha-Diago, Miguel

Perepelyuk, Maryna

Petridis, Lambis

Piluso, Susanna

Pistone, Alessandro

Pucci, Andrea

Quencer, Keith

Radecki, Jerzy

Ranzato, Elia

Ravaioli, Stefano

Ren, Yanfang

Rohnke, Marcus

Roldo, Marta

Rossouw, Paul E.

Santos, Joao

Sebastian, Aimy

Seeliger, Claudine

Sgarbossa, Paolo

Sharma, Bhesh

Shavandi, Amin

Shie, Ming-You

Shih, Yu-Ru

Silikas, Nikolaos

Silva, Simone S.

Simoni, Jan

Siracusa, Valentina

Spriano, Silvia

Stamboulis, Artemis

Stan, George

Surmenev, Roman A.

Swaminathan, Viswanathan

Takeshita, Haruo

Taubenberger, Anna

Tavri, Sidhartha

(C) 2018 by the authors. Licensee MDPI, Basel, Switzerland. This article is an open access article distributed under the terms and conditions of the Creative Commons Attribution (CC BY) license (http://creativecommons.org/licenses/by/4.0/). 